\title{
Trace FeCu@PC derived from MOFs for ultra-efficient heterogeneous electro-Fenton process: Enhanced electron transfer and bimetallic synergy
}

\section{Supporting information}

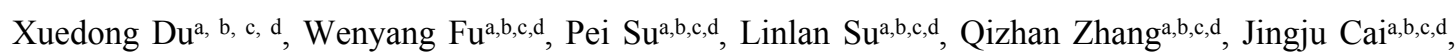
Minghua Zhou a, b, c, d*

${ }^{a}$ Key Laboratory of Pollution Process and Environmental Criteria, Ministry of Education, College of Environmental Science and Engineering, Nankai University, Tianjin, 300350, China

${ }^{b}$ Tianjin Key Laboratory of Environmental Technology for Complex Trans-Media Pollution, Nankai University, Tianjin, 300350, China

${ }^{c}$ Tianjin Key Laboratory of Urban Ecology Environmental Remediation and Pollution Control, College of Environmental Science and Engineering, Nankai University, Tianjin, 300350, China

${ }^{d}$ Tianjin Advanced Water Treatment Technology International Joint Research Center, College of Environmental Science and Engineering, Nankai University, Tianjin, 300350, China

*Corresponding author. Tel.: +86 022 23501117; fax: +86 02223501117.

E-mail address: zhoumh@nankai.edu.cn (M. Zhou). 


\section{Contents}

\section{Texts}

Text S1. Preparation of modified carbon felt

Text S2. HPLC and UPLC-HRMS methods

Text S3. Current efficiency

Text S4. Mineralization current efficiency

Text S5. DFT Calculation method

Text S6. Partial catalytic mechanism equations

\section{Tables}

Table S1. Textural properties of FeCu@PC-800/6.

Table S2. Parameters of the pseudo-first order rate constant for SMT degradation in Fe@PC/EF, FeCu@PC/EF, and Cu@PC/EF.

Table S3. Summary of some relevant studies on Fenton/Fenton-like system catalyzed by FeCu bimetal based solid catalysts.

Table S4. SMT and intermediates products generated during the degradation of SMT in $\mathrm{FeCu} @ \mathrm{PC}-$ 800/6/Hetero-EF system.

Table S5. Quality parameters of the secondary wastewater effluent.

\section{Figures}

Fig. S1. XRD patterns of Fe-MOFs, Fe/Cu-MOFs, and Cu-MOFs.

Fig. S2. XRD patterns of $\mathrm{Fe} @ \mathrm{PC}, \mathrm{FeCu} @ \mathrm{PC}$, and $\mathrm{Cu} @ \mathrm{PC}$ under different pyrolysis temperature: (a) $400{ }^{\circ} \mathrm{C}$, (b) $500{ }^{\circ} \mathrm{C}$, (c) $550{ }^{\circ} \mathrm{C}$, (d) $600{ }^{\circ} \mathrm{C}$, (e) $700{ }^{\circ} \mathrm{C}$, and (f) $800{ }^{\circ} \mathrm{C}$.

Fig. S3. SEM images of (a) Fe@PC-800/6 and (b) Cu@PC-800/6; TEM images of (c) Fe@PC-800/6 and (d) Cu@PC-800/6. (e) N 1s core-level spectra of FeCu@PC-800/6. (f) Cu LMM AES spectrum of FeCu@PC-800/6.

Fig. S4. Effect of current intensity on $\mathrm{H}_{2} \mathrm{O}_{2}$ production. Conditions: $200.0 \mathrm{~mL}, 0.05 \mathrm{M} \mathrm{Na}_{2} \mathrm{SO}_{4}, \mathrm{pH} 4.0$ The inset pattern is a photograph of the current efficiency of $\mathrm{H}_{2} \mathrm{O}_{2}$ production. 
Fig. S5. The effect of various parameters on the SMT degradation in the FeCu@PC-800/6/Hetero-EF process: (a) initial pH, (b) current, (c) catalyst concentration, and (d) SMT concentration. Except for the investigated parameter, other parameters were set as follows: $\mathrm{pH}, 4.0$; current, $25.0 \mathrm{~mA}$; catalyst, 25.0 $\mathrm{mg} \mathrm{L} \mathrm{L}^{-1}$; initial SMT, $10.0 \mathrm{mg} \mathrm{L}^{-1}$.

Fig. S6. The kinetics analysis as a pseudo-first-order reaction. (a) Comparison of the catalytic performance under different conditions. The effect of various parameters on the SMT degradation in the FeCu@PC-800/6/Hetero-EF process: (b) initial pH, (c) current, (d) catalyst concentration, and (e) SMT concentration.

Fig. S7. Effect of scavengers: $300 \mathrm{mM} n$-butanol, $3000 \mathrm{U} \mathrm{L}^{-1} \mathrm{SOD}$, and $10 \mathrm{mM}$ KI. Conditions: pH, 4.0; current, $25.0 \mathrm{~mA}$; initial SMT, $10.0 \mathrm{mg} \mathrm{L}^{-1}$; catalyst, $25.0 \mathrm{mg} \mathrm{L}^{-1}$

Fig. S8. Top-view and side-view structures of FeCu@PC-800/6 (a, c) and Fe@PC-800/6 (b, d).

Fig. S9. HOMO and LUMO spatial distribution of PC (a,d), Fe@PC-800/6 (b,e) and FeCu@PC-800/6 (c,f) respectively.( vaspmo03 and mutiwfn software deal with this picture, and green and blue regions represents the positive and negative electron cloud, respectively.)

Fig. S10. The model of hydrated ion.

Fig. S11. The reaction energies of $\mathrm{Cu}^{+}$reducing $\mathrm{Fe}^{3+}$ in solution.

Fig. S12. Evolution of carboxylic acids formed in the FeCu@PC-800/6/Hetero-EF process. Conditions: initial SMT, $50.0 \mathrm{mg} \mathrm{L}^{-1}$; $\mathrm{pH}, 4.0$; catalyst, $50.0 \mathrm{mg} \mathrm{L}^{-1}$; current, $25.0 \mathrm{~mA}$.

Fig. S13. (a) Consecutive runs to probe the evolution of catalytic activity for $\mathrm{FeCu} @ \mathrm{PC}-800 / 6$ as well as the corresponding iron and copper ions concentration. (For the cycling tests, the catalyst was recycled by external magnetic field, and then directly reused for the next run without washing. The thermal treatment on the recycled catalyst at $400{ }^{\circ} \mathrm{C}$ for $1 \mathrm{~h}$ in $\mathrm{H}_{2}$ with the heating rate of $10^{\circ} \mathrm{C} \mathrm{min}^{-1}$ was applied to regenerate the catalytic activity before each run.) Conditions: $\mathrm{pH}, 4.0$; current, $25.0 \mathrm{~mA}$; initial SMT, $10.0 \mathrm{mg} \mathrm{L}^{-1}$; catalyst, $25.0 \mathrm{mg} \mathrm{L}-1$. (b) XRD pattern of pristine, used and regenerated $\mathrm{FeCu} @ \mathrm{PC}-800 / 6$. Fig. S14. (a) Degradation of rhodamine B, 2,4-dichlorophenoxyacetic acid, carbamazepine, and phenol, respectively, in the $\mathrm{FeCu} @ \mathrm{PC}-800 / 6 /$ Hetero-EF process. Conditions: pH, 4.0; current, $25.0 \mathrm{~mA}$; pollutant, $10.0 \mathrm{mg} \mathrm{L}^{-1}$; catalyst, $25.0 \mathrm{mg} \mathrm{L}^{-1}$. (b) Degradation of the mixture of rhodamine $\mathrm{B}, 2,4-$ dichlorophenoxyacetic acid, carbamazepine, and phenol in the $\mathrm{FeCu} @ \mathrm{PC}-800 / 6 / \mathrm{Hetero}-\mathrm{EF}$ process. Conditions: $\mathrm{pH}, 4.0$; current, $25.0 \mathrm{~mA}$; each pollutant, $1.0 \mathrm{mg} \mathrm{L}-1$; catalyst, $25.0 \mathrm{mg} \mathrm{L}^{-1}$. 
Fig. S15. Effect of background constituents: (a) $\mathrm{NaCl}$, (b) $\mathrm{NaHCO}_{3}$, (c) $\mathrm{NaNO}_{3}$ and (d) humic acid. Conditions: pH, 4.0; current, $25.0 \mathrm{~mA}$; SMT, $10.0 \mathrm{mg} \mathrm{L}^{-1}$; catalyst, $25.0 \mathrm{mg} \mathrm{L} \mathrm{L}^{-1}$.

Fig. S16. Degradation of SMT in the simulated wastewater, secondary wastewater or industrial wastewater effluent by the FeCu@PC-800/6/Hetero-EF process. Conditions: pH, 4.0; current, 25.0 mA; SMT, $10.0 \mathrm{mg} \mathrm{L}^{-1}$; catalyst, $25.0 \mathrm{mg} \mathrm{L}^{-1}$.

Fig. S17. (a) Consecutive runs to probe the evolution of catalytic activity for $\mathrm{FeCu} @ \mathrm{PC}-800 / 6$ and $\mathrm{Fe}^{0}$ in the secondary wastewater effluent. (For cycling tests, the catalyst was recycled by external magnetic field, and then directly reused for the next run without washing.) (b) After regeneration, probe the evolution of catalytic activity for FeCu@PC-800/6 and Fe $\mathrm{Fe}^{0}$ in the secondary wastewater effluent. (The thermal treatment on the recycled catalyst at $400{ }^{\circ} \mathrm{C}$ for $1 \mathrm{~h}$ in $\mathrm{H}_{2}$ with the heating rate of $10{ }^{\circ} \mathrm{C} \min ^{-1}$ was applied to regenerate the catalytic activity before each run.) Conditions: $\mathrm{pH}, 4.0$; current, $25.0 \mathrm{~mA}$; SMT, $10.0 \mathrm{mg} \mathrm{L}^{-1}$; catalyst, $25.0 \mathrm{mg} \mathrm{L}^{-1}$. 


\section{Texts}

Text S1. Preparation of modified carbon felt

The carbon felt modified with carbon black with a geometric area of $5.0 \mathrm{~cm}^{2}(2.5 \mathrm{~cm} \times 2.0 \mathrm{~cm})$ was used as the cathode for the electrochemical generation of $\mathrm{H}_{2} \mathrm{O}_{2}$. The electrode was prepared using carbon black $(0.10 \mathrm{~g})$ as catalyst, PTFE $(0.18 \mathrm{~mL})$ as hydrophobic binder, and water/EtOH $(1: 1 \mathrm{v} / \mathrm{v}, 1.40 \mathrm{~mL})$ as solvent. The above mixture paste was coated on two side of carbon felt. After drying in air, the modified carbon felt was annealed at $360{ }^{\circ} \mathrm{C}$ with the heating rate of $10{ }^{\circ} \mathrm{C} \mathrm{min}-1$ under air atmosphere for $30 \mathrm{~min}$ to form the three-dimensional structure.

Text S2. HPLC and UPLC-HRMS methods

The SMT concentration was determined by high performance liquid chromatography (HPLC, U3000, Thermo Fisher Scientific) with a DAD detector at the wavelength of $270 \mathrm{~nm}$ and an Acclaim ${ }^{\mathrm{TM}}$ $120 \mathrm{C} 18$ column $(3 \mu \mathrm{m}, \varphi 3.0 \times 100 \mathrm{~mm})$. The mobile phase was composed of water and ACN $(65: 35$, $\mathrm{v} / \mathrm{v}$ ) at a flow rate of $0.3 \mathrm{~mL} \mathrm{~min}{ }^{-1}$ with a column temperature of $30^{\circ} \mathrm{C}$. The intermediates of SMT were concentrated by solid phase extraction (SPE) using hydrophilic-liphophilic balance (HLB) (Waters Oasis) cartridges. Degradation products were determined by UPLC (UltiMate 3000, Thermo Fisher Scientific) with a Syncronis C18 column $(1.7 \mu \mathrm{m}, \varphi 2.1 \times 100 \mathrm{~mm})$ coupled to orbitrap mass spectrometer (HRMS, Orbitrap Fusion, Thermo Fisher Scientific). The gradient mobile phase was the combination of water (A) and $\mathrm{ACN}(\mathrm{B})$ both containing $0.1 \%(\mathrm{v} / \mathrm{v})$ formic acid. The gradient elution was programmed as follows: 0.0-7.0 $\min , 5-90 \% \mathrm{~B}$; 7.0-9.5 $\min , 90 \% \mathrm{~B}$; 9.5-9.6 $\min , 90-5 \% \mathrm{~B}, 9.6-13.0 \mathrm{~min}, 5 \% \mathrm{~B}$. Mass analyzer was operated in full scan mode $(\mathrm{m} / \mathrm{z}$ range 50-500) in order to identify the products.

Text S3. Current efficiency

The current efficiency (CE) could be calculated through the following equation:

$$
C E=\frac{n F C V}{\int_{0}^{t} I d t} \times 100 \%
$$


where $n$ is the number of electrons transferred for oxygen reduction to $\mathrm{H}_{2} \mathrm{O}_{2}, \mathrm{~F}$ is the Faraday constant (96,486 $\left.\mathrm{C} \mathrm{mol}^{-1}\right), C$ is the concentration of $\mathrm{H}_{2} \mathrm{O}_{2}$ formed (mol L-1), $V$ is the bulk volume (L), $I$ is the current (A), and $t$ is the time (s).

Text S4. Mineralization current efficiency

The mineralization current efficiency (MCE) could be calculated through the following equation:

$$
\operatorname{MCE}(\%)=\frac{n F V \Delta(T O C)_{\text {exp }}}{4.32 \times 10^{7} m I t} \times 100
$$

Where $n$ is the electrons consumed per SMT molecule during its mineralization was taken as 52, $F$ is the Faraday constant $\left(96,487 \mathrm{C} \mathrm{mol}^{-1}\right), V_{s}$ is the bulk volume (L), $\Delta(T O C)_{\exp }$ is the TOC decay $\left(\mathrm{mg} \mathrm{L}^{-1}\right), 4.32$ $\times 10^{7}$ is the conversion factor $\left(3600 \mathrm{~s} / \mathrm{h} \times 1200 \mathrm{mg} \mathrm{C} \mathrm{mol}^{-1}\right), m$ represents the number of carbon atoms of SMT (12), $I$ is current (I) and $t$ represents time (h).

\section{Text S5. DFT Calculation method}

The Vienna ab initio Simulation Package (VASP, Version 5.4.1) was used to determine geometry structure optimization, density of state (DOS), and band gap. ${ }^{1}$ Using Local density approximation with Projector-augmented wave (PAW) pseudopotentials to obtain the exchangecorrelation energy. The cutoff energy was set as $400 \mathrm{eV}$, and structure relaxation was performed until the convergence criteria of force reached $0.03 \mathrm{eV} \AA^{-1} \cdot \mathrm{Fe}^{0}$ and $\mathrm{Cu}^{0}$ was simulated by a single atom, ${ }^{2-3}$ and graphite (002) constructed the PC based on the XRD characterization. Thus, the model of FeCu@PC-800/6 was constructed by using that both Fe atom and $\mathrm{Cu}$ atom loaded on the graphite (002) with the lattice parameters of $\mathrm{a}=9.84 \AA, \mathrm{b}=9.84 \AA$ and $\mathrm{c}=18.4 \AA$ (Fig. S1). Similarly, the model of Fe@PC-800/6 was that Fe atom individually loaded on the graphite (002) with the same lattice parameters. The Brillouin zone was sampled with $1 \times 1 \times 1$ and $7 \times 7 \times 3 \mathrm{~K}$-points grids for geometry structure optimization and electronic property, respectively.

The $\mathrm{Fe}^{3+}, \mathrm{Fe}^{2+}, \mathrm{Cu}^{2+}$ and $\mathrm{Cu}^{+}$can be modeled by hydrated ion in Fig. S2. The Gibbs free energy of $\mathrm{Cu}^{+}$reducing $\mathrm{Fe}^{3+}$ can be calculated by (Eq. S3):

$$
\Delta \mathrm{G}=\mathrm{E}\left(\mathrm{Fe}^{2+}\right)+\mathrm{E}\left(\mathrm{Cu}^{2+}\right)-\mathrm{E}\left(\mathrm{Fe}^{3+}\right)-\mathrm{E}\left(\mathrm{Cu}^{+}\right)+\mathrm{EZPT}-\mathrm{T} \Delta \mathrm{S}
$$

Where E, T, E $\mathrm{E}_{\mathrm{ZPT}}$ and $\Delta \mathrm{S}$ are energy from DFT calculation, reaction temperature, zero point energy and the change of entropy in reaction, respectively. 
Text S6. Partial catalytic mechanism equations

$$
\begin{aligned}
& \mathrm{Fe}^{2+}+\mathrm{H}_{2} \mathrm{O}_{2}+\mathrm{H}^{+} \rightarrow \mathrm{Fe}^{3+}+\mathrm{OH}^{\text {free }}+\mathrm{H}_{2} \mathrm{O} \\
& \mathrm{Fe}^{3+}+\mathrm{H}_{2} \mathrm{O}_{2} \rightarrow \mathrm{Fe}^{2+}+\mathrm{HO}_{2} \text { free }+\mathrm{H}^{+} \\
& \mathrm{Cu}^{+}+\mathrm{H}_{2} \mathrm{O}_{2}+\mathrm{H}^{+} \rightarrow \equiv \mathrm{Cu}^{2+}+\mathrm{OH}^{\text {free }}+\mathrm{H}_{2} \mathrm{O} \\
& \mathrm{Cu}^{2+}+\mathrm{H}_{2} \mathrm{O}_{2} \rightarrow \mathrm{Cu}^{+}+\mathrm{HO}_{2} \text { free } \\
& \mathrm{OH}^{+} \\
& \mathrm{OH}^{\text {free }}+\mathrm{SMT} \rightarrow \text { byproducts } \rightarrow \mathrm{CO}_{2}+\mathrm{H}_{2} \mathrm{O} \\
& \mathrm{Cu}^{+}+\mathrm{Fe}^{3+} \rightarrow \mathrm{Cu}^{2+}+\mathrm{Fe}^{2+}
\end{aligned}
$$


Table S1. Textural properties and surface elemental contents of FeCu@PC-800/6.

\begin{tabular}{cccccccc}
\hline Sample & \multirow{2}{*}{$\begin{array}{c}S_{\mathrm{BET}} \\
\left(\mathrm{m}^{2} \mathrm{~g}^{-1}\right)\end{array}$} & $\begin{array}{c}\text { Total pore volume } \\
\left(\mathrm{cm}^{3} \mathrm{~g}^{-1}\right)\end{array}$ & Average pore & \multicolumn{4}{c}{ Surface atomic content } \\
\cline { 5 - 8 } & 66.8 & 0.15 & diameter $(\mathrm{nm})$ & $\mathrm{C}(\%)$ & $\mathrm{O}(\%)$ & $\mathrm{Fe} \mathrm{( \% )}$ & $\mathrm{Cu}(\%)$ \\
\hline $\mathrm{FeCu} @ \mathrm{PC}-800 / 6$ & 66.8 & 12.8 & 81.4 & 12.4 & 4.1 & 2.1 \\
\hline
\end{tabular}


Table S2. Parameters of the pseudo-first order kinetic model for SMT degradation in Fe@PC/HeteroEF, FeCu@PC/Hetero-EF, and Cu@PC/Hetero-EF.

\begin{tabular}{|c|c|c|c|c|c|c|c|}
\hline \multirow{2}{*}{ Catalysts } & \multicolumn{2}{|c|}{ Model parameters } & \multirow{2}{*}{$f$} & \multirow{2}{*}{ Catalysts } & \multicolumn{2}{|c|}{ Model parameters } & \multirow{2}{*}{$f$} \\
\hline & $k\left(\min ^{-1}\right)$ & $R^{2}$ & & & $k\left(\min ^{-1}\right)$ & $R^{2}$ & \\
\hline $\mathrm{Fe} @ \mathrm{C}-400 / 2$ & 0.012 & 0.9909 & & $\mathrm{Fe} @ \mathrm{C}-600 / 2$ & 0.028 & 0.9949 & \\
\hline $\mathrm{FeCu} @ \mathrm{C}-400 / 2$ & 0.019 & 0.9977 & 0.731 & $\mathrm{FeCu} @ \mathrm{C}-600 / 2$ & 0.050 & 0.9919 & 1.250 \\
\hline $\mathrm{Cu} @ \mathrm{C}-400 / 2$ & 0.014 & 0.9915 & & $\mathrm{Cu} @ \mathrm{C}-600 / 2$ & 0.012 & 0.9939 & \\
\hline $\mathrm{Fe} @ \mathrm{C}-400 / 6$ & 0.008 & 0.9979 & & $\mathrm{Fe} @ \mathrm{C}-600 / 6$ & 0.026 & 0.9883 & \\
\hline $\mathrm{FeCu} @ \mathrm{C}-400 / 6$ & 0.015 & 0.9979 & 0.882 & FeCu@C-600/6 & 0.061 & 0.9946 & 1.694 \\
\hline $\mathrm{Cu} @ \mathrm{C}-400 / 6$ & 0.009 & 0.9987 & & $\mathrm{Cu} @ \mathrm{C}-600 / 6$ & 0.010 & 0.9806 & \\
\hline $\mathrm{Fe} @ \mathrm{C}-500 / 2$ & 0.011 & 0.9876 & & $\mathrm{Fe} @ \mathrm{C}-700 / 2$ & 0.033 & 0.9824 & \\
\hline FeCu@C-500/2 & 0.015 & 0.9980 & 0.714 & FeCu@C-700/2 & 0.053 & 0.9807 & 1.262 \\
\hline $\mathrm{Cu} @ \mathrm{C}-500 / 2$ & 0.010 & 0.9925 & & $\mathrm{Cu} @ \mathrm{C}-700 / 2$ & 0.009 & 0.9990 & \\
\hline $\mathrm{Fe} @ \mathrm{C}-500 / 6$ & 0.008 & 0.9952 & & $\mathrm{Fe} @ \mathrm{C}-700 / 6$ & 0.046 & 0.9904 & \\
\hline FeCu@C-500/6 & 0.009 & 0.9938 & 0.529 & FeCu@C-700/6 & 0.074 & 0.9916 & 1.321 \\
\hline $\mathrm{Cu} @ \mathrm{C}-500 / 6$ & 0.009 & 0.9971 & & Cu@C-700/6 & 0.010 & 0.9977 & \\
\hline $\mathrm{Fe} @ \mathrm{C}-550 / 2$ & 0.011 & 0.9923 & & $\mathrm{Fe} @ \mathrm{C}-800 / 2$ & 0.032 & 0.9808 & \\
\hline FeCu@C-550/2 & 0.021 & 0.9732 & 0.875 & $\mathrm{FeCu} @ \mathrm{C}-800 / 2$ & 0.064 & 0.9910 & 1.524 \\
\hline $\mathrm{Cu} @ \mathrm{C}-550 / 2$ & 0.013 & 0.9900 & & $\mathrm{Cu} @ \mathrm{C}-800 / 2$ & 0.010 & 0.9944 & \\
\hline $\mathrm{Fe} @ \mathrm{C}-550 / 6$ & 0.012 & 0.9960 & & $\mathrm{Fe} @ \mathrm{C}-800 / 6$ & 0.030 & 0.9792 & \\
\hline FeCu@C-550/6 & 0.033 & 0.9639 & 1.375 & FeCu@C-800/6 & 0.098 & 0.9948 & 1.815 \\
\hline $\mathrm{Cu} @ \mathrm{C}-550 / 6$ & 0.012 & 0.9946 & & $\mathrm{Cu} @ \mathrm{C}-800 / 6$ & 0.024 & 0.9694 & \\
\hline
\end{tabular}


Table S3. Summary of some relevant studies on Fenton/Fenton-like system catalyzed by FeCu bimetal based solid catalysts.

\begin{tabular}{|c|c|c|c|c|c|c|}
\hline Catalyst & Technology & Operating conditions & $\begin{array}{l}\text { Reaction } \\
\text { time (min) }\end{array}$ & $\begin{array}{l}\text { Removal } \\
\text { efficiency (\%) }\end{array}$ & $\begin{array}{c}\mathrm{TOF}_{\mathrm{d}} \\
\left(\mathrm{L} \mathrm{g}^{-1} \min ^{-1}\right)\end{array}$ & Ref \\
\hline $\mathrm{CuFeO}_{2}$ & Fenton & $\begin{array}{l}\text { BPA } 0.1 \mathrm{mmol} \mathrm{L}^{-1} \text {, catalyst } 1.0 \mathrm{~g} \mathrm{~L}^{-1} \text {, } \\
\mathrm{H}_{2} \mathrm{O}_{2} 0.02 \mathrm{~mol} \mathrm{~L}^{-1}, \mathrm{pH} 5.0\end{array}$ & 120 & 100 & 0.05 & 4 \\
\hline $\mathrm{Cu}$-doped goethite & Fenton & $\begin{array}{l}\text { Quinoline } 10 \mathrm{mg} \mathrm{L}^{-1} \text {, catalyst } 1.0 \mathrm{~g} \mathrm{~L}^{-} \\
{ }^{1}, \mathrm{H}_{2} \mathrm{O}_{2} 0.17 \mathrm{~mol} \mathrm{~L}^{-1}\end{array}$ & 120 & 50 (TOC) & & 5 \\
\hline $\mathrm{ZVI} / \mathrm{Cu}$ & Fenton & $\begin{array}{l}\text { Orange II } 25 \mathrm{mg} \mathrm{L}^{-1} \text {, catalyst } 0.05 \mathrm{~g} \mathrm{~L}^{-} \\
{ }^{1} \text {, air } 0.8 \mathrm{~L} \mathrm{~min}^{-1}, \mathrm{pH} 3.0\end{array}$ & 50 & 100 & & 6 \\
\hline $\mathrm{CuFe} / \mathrm{SBA}-15$ & Fenton & $\begin{array}{l}\text { DPD } 1.0 \mathrm{~g} \mathrm{~L}^{-1} \text {, catalyst } 0.1 \mathrm{~g} \mathrm{~L}^{-1}, \mathrm{H}_{2} \mathrm{O}_{2} \\
0.08 \mathrm{~mol} \mathrm{~L}^{-1}, \mathrm{pH} 4.0\end{array}$ & 120 & 83 & 0.35 & 7 \\
\hline Fe-Cu@MPSi & Fenton & $\begin{array}{l}\text { OFL } 30 \mathrm{mg} \mathrm{L}^{-1} \text {, catalyst } 1.0 \mathrm{~g} \mathrm{~L}^{-1} \text {, } \\
\mathrm{H}_{2} \mathrm{O}_{2} 2.0 \mathrm{~g} \mathrm{~L}^{-1}, \mathrm{pH} 3.0\end{array}$ & 120 & 85 & & 8 \\
\hline 2Fe6Cu/HMS & Fenton & $\begin{array}{l}\text { Orange II } 100 \mathrm{mg} \mathrm{L}^{-1} \text {, catalyst } 1.0 \mathrm{~g} \mathrm{~L}- \\
{ }^{1}, \mathrm{H}_{2} \mathrm{O}_{2} 0.03 \mathrm{~mol} \mathrm{~L}^{-1}, \mathrm{pH} 7.0\end{array}$ & 120 & 93 & & 9 \\
\hline Fe-Cu@Al-MCM-41 & Fenton & $\begin{array}{l}\text { Phenol } 200 \mathrm{mg} \mathrm{L}^{-1} \text {, catalyst } 1.5 \mathrm{~g} \mathrm{~L}^{-1} \text {, } \\
\mathrm{H}_{2} \mathrm{O}_{2} 0.05 \mathrm{~mol} \mathrm{~L}^{-1}, \mathrm{pH} 4.0\end{array}$ & 120 & 47 (TOC) & & 10 \\
\hline $\begin{array}{l}\gamma-\mathrm{Fe}_{2} \mathrm{O}_{3} @ \mathrm{Cu} / \mathrm{Al}-\mathrm{MCM}- \\
41\end{array}$ & Fenton & $\begin{array}{l}\text { Phenol } 80 \mathrm{mg} \mathrm{L}^{-1} \text {, catalyst } 1.0 \mathrm{~g} \mathrm{~L}^{-1} \text {, } \\
\mathrm{H}_{2} \mathrm{O}_{2} 0.05 \mathrm{~mol} \mathrm{~L}^{-1}, \mathrm{pH} 4.0\end{array}$ & 120 & 64 (TOC) & & 11 \\
\hline $5 \mathrm{Fe} 2.5 \mathrm{Cu}-\mathrm{Al}_{2} \mathrm{O}_{3}$ & Fenton & $\begin{array}{l}\text { NB } 100 \mathrm{mg} \mathrm{L}^{-1} \text {, catalyst } 1.0 \mathrm{~g} \mathrm{~L}^{-1} \text {, } \\
\mathrm{H}_{2} \mathrm{O}_{2} 0.3 \mathrm{~mL}(30 \%), \mathrm{pH} 3.0\end{array}$ & 60 & 100 & & 12 \\
\hline CuFeZSM-5 & Fenton & $\begin{array}{l}\text { R6G } 100 \mathrm{mg} \mathrm{L}^{-1} \text {, catalyst } 1.0 \mathrm{~g} \mathrm{~L}^{-1} \text {, } \\
\mathrm{H}_{2} \mathrm{O}_{2} 0.04 \mathrm{~mol} \mathrm{~L}^{-1}, \mathrm{pH} 3.4\end{array}$ & 40 & 100 & & 13 \\
\hline $\mathrm{CuFe}-\mathrm{MC}$ & Fenton & $\begin{array}{l}\text { BPA } 100 \mathrm{mg} \mathrm{L}^{-1} \text {, catalyst } 0.3 \mathrm{~g} \mathrm{~L}^{-1} \text {, } \\
\mathrm{H}_{2} \mathrm{O}_{2} 0.03 \mathrm{~mol} \mathrm{~L}^{-1}, \mathrm{pH} 3.0\end{array}$ & 60 & 93 & 0.15 & 14 \\
\hline $\mathrm{FeCu} @ \mathrm{C}$ & Fenton & $\begin{array}{l}\text { SMT } 20 \mathrm{mg} \mathrm{L}^{-1} \text {, catalyst } 0.25 \mathrm{~g} \mathrm{~L}^{-1} \text {, } \\
\mathrm{H}_{2} \mathrm{O}_{2} 1.5 \mathrm{mmol} \mathrm{L}^{-1} \text {, pH } 3.0\end{array}$ & 60 & 90 & & 15 \\
\hline $\mathrm{Cu}$-doped $\alpha$-FeOOH & Photo-Fenton & $\begin{array}{l}\text { DCF } 40 \mathrm{mg} \mathrm{L}^{-1} \text {, catalyst } 0.4 \mathrm{~g} \mathrm{~L}^{-1} \text {, } \\
\mathrm{H}_{2} \mathrm{O}_{2} 0.1 \mathrm{~mol} \mathrm{~L}^{-1}, \mathrm{pH} 6.1 \text {, Vis }\end{array}$ & 60 & 95 & & 16 \\
\hline $\mathrm{Fe}_{3} \mathrm{O}_{4} / \mathrm{C} / \mathrm{Cu}$ & Photo-Fenton & $\begin{array}{l}\text { MB } 100 \mathrm{mg} \mathrm{L}^{-1} \text {, catalyst } 0.5 \mathrm{~g} \mathrm{~L}^{-1} \text {, } \\
\mathrm{H}_{2} \mathrm{O}_{2} 0.16 \mathrm{~mol} \mathrm{~L}^{-1}, \mathrm{pH} 6.9 \text {, Vis }\end{array}$ & 80 & 100 & & 17 \\
\hline $\mathrm{FeCuC}$ aerogel & Electro-Fenton & $\begin{array}{l}\text { MB } 50 \mathrm{mg} \mathrm{L}^{-1} \text {, cathode, } 2 \mathrm{~cm}^{2} \text {, current } \\
10 \mathrm{~mA}, \mathrm{pH} 3\end{array}$ & 30 & 98 & & 18 \\
\hline $\mathrm{Fe}_{2.75} \mathrm{Cu}_{0.25} \mathrm{O}_{4}$ & Electro-Fenton & AM $100 \mathrm{mg} \mathrm{L}^{-1}$, catalyst $0.088 \mathrm{~g} \mathrm{~L}^{-1}$, & 90 & 98 & 0.48 & 19 \\
\hline
\end{tabular}




\begin{tabular}{|c|c|c|c|c|c|c|}
\hline & & potential -1.1 vs $\mathrm{Ag} / \mathrm{AgCl}$, pH 13.4 & & & & \\
\hline $\mathrm{FeCu} @ \mathrm{PC}-800 / 6$ & Electro-Fenton & $\begin{array}{l}\text { SMT } 10 \mathrm{mg} \mathrm{L}^{-1} \text {, catalyst } 0.025 \mathrm{~g} \mathrm{~L}^{-1} \text {, } \\
\text { current } 25 \mathrm{~mA}, \mathrm{pH} 4\end{array}$ & 60 & 99 & 3.92 & $\begin{array}{l}\text { This } \\
\text { work }\end{array}$ \\
\hline $\mathrm{FeCu} @ \mathrm{PC}-800 / 6$ & Electro-Fenton & $\begin{array}{l}\text { SMT } 20 \mathrm{mg} \mathrm{L}^{-1} \text {, catalyst } 0.025 \mathrm{~g} \mathrm{~L}^{-1} \text {, } \\
\text { current } 25 \mathrm{~mA}, \mathrm{pH} 4\end{array}$ & 60 & 90 & 1.72 & $\begin{array}{l}\text { This } \\
\text { work }\end{array}$ \\
\hline
\end{tabular}

Note: ZVI, Zero-valent iron; MPSi, mesoporous silicon; HMS, hollow mesoporous silica spheres; Vis, visible light ( $\lambda>420 \mathrm{~nm})$; BPA, bisphenol A; DPD, N,Ndiethyl-p-phenyl diamine; OFL, ofloxacin; NB, nitrobenzene; R6G, Rhodamine 6G; DCF, diclofenac sodium; MB, methylene blue; AM, amaranth

Table S4. SMT and intermediates products generated during the degradation of SMT in $\mathrm{FeCu} @ \mathrm{PC}-$ 800/6/Hetero-EF system.

\begin{tabular}{|c|c|c|c|c|c|}
\hline Compound & $\begin{array}{l}\text { Chemical } \\
\text { formula }\end{array}$ & $\begin{array}{l}\text { Retention } \\
\text { time } \\
\text { (min) }\end{array}$ & Molecular structure & $\begin{array}{l}\text { Molecular } \\
\text { mass } \\
\left(\mathrm{g} \mathrm{mol}^{-1}\right)\end{array}$ & $m / z$ \\
\hline $\begin{array}{l}\text { Sulfamethazine } \\
\qquad \text { (SMT) }\end{array}$ & $\mathrm{C}_{12} \mathrm{H}_{14} \mathrm{~N}_{4} \mathrm{O}_{2} \mathrm{~S}$ & 3.17 & & 278.08 & $\begin{array}{c}279.09 \\
\left(\mathrm{M}^{+}\right)\end{array}$ \\
\hline$N^{4}$-OH-SMT & $\mathrm{C}_{12} \mathrm{H}_{14} \mathrm{~N}_{4} \mathrm{O}_{3} \mathrm{~S}$ & 2.81 & & 294.08 & $\begin{array}{c}295.08 \\
\left(\mathrm{M}^{+}\right)\end{array}$ \\
\hline 4-NO-SMT & $\mathrm{C}_{12} \mathrm{H}_{12} \mathrm{~N}_{4} \mathrm{O}_{3} \mathrm{~S}$ & 2.38 & & 292.06 & $\begin{array}{c}293.07 \\
\left(\mathrm{M}^{+}\right)\end{array}$ \\
\hline $\begin{array}{l}\text { 4-(2-Imino-4,6- } \\
\text { dimethylpyrimidin- } \\
\text { 1(2H)-yl) aniline }\end{array}$ & $\mathrm{C}_{12} \mathrm{H}_{14} \mathrm{~N}_{4}$ & 1.83 & & 214.12 & $\begin{array}{c}215.13 \\
\left(\mathrm{M}^{+}\right)\end{array}$ \\
\hline $\begin{array}{l}\text { 4-(2-Imino-4,6- } \\
\text { dimethylpyrimidin- } \\
\text { 1(2H)-yl) nitroso- } \\
\text { benzene }\end{array}$ & $\mathrm{C}_{12} \mathrm{H}_{12} \mathrm{~N}_{4} \mathrm{O}$ & 2.02 & & 228.10 & $\begin{array}{c}229.11 \\
\left(\mathrm{M}^{+}\right)\end{array}$ \\
\hline $\begin{array}{c}\text { 2-Amino-4,6- } \\
\text { dimethylpyrimidine }\end{array}$ & $\mathrm{C}_{6} \mathrm{H}_{9} \mathrm{~N}_{3}$ & 0.69 & & 123.08 & $\begin{array}{c}124.09 \\
\left(\mathrm{M}^{+}\right)\end{array}$ \\
\hline Sulfanilic acid & $\mathrm{C}_{6} \mathrm{H}_{7} \mathrm{NO}_{3} \mathrm{~S}$ & 1.97 & & 173.01 & $\begin{array}{c}172.01 \\
\left(\mathrm{M}^{-}\right)\end{array}$ \\
\hline $\begin{array}{l}\text { 4-Hydroxyamino- } \\
\text { benzenesulfonic } \\
\text { acid }\end{array}$ & $\mathrm{C}_{6} \mathrm{H}_{7} \mathrm{NO}_{4} \mathrm{~S}$ & 1.54 & 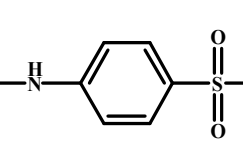 & 189.01 & $\begin{array}{c}188.00 \\
\left(\mathrm{M}^{-}\right)\end{array}$ \\
\hline $\begin{array}{l}\text { 4-Amino-N- } \\
\text { carbamimidoyl- } \\
\text { benzenesulfanamide }\end{array}$ & $\mathrm{C}_{7} \mathrm{H}_{10} \mathrm{~N}_{4} \mathrm{O}_{2} \mathrm{~S}$ & 2.49 & & 214.05 & $\begin{array}{c}215.06 \\
\left(\mathrm{M}^{+}\right)\end{array}$ \\
\hline
\end{tabular}


Table S5. Quality parameters of the secondary wastewater effluent and industrial wastewater effluent.

\begin{tabular}{ccc}
\hline & Secondary wastewater effluent & Industrial wastewater effluent \\
\hline $\mathrm{pH}$ & 7.54 & 8.18 \\
Conductivity $\left(\mu \mathrm{S} \mathrm{cm}^{-1}\right)$ & 820 & 4890 \\
$\mathrm{HCO}_{3}^{-}\left(\mathrm{mg} \mathrm{L}^{-1}\right)$ & 43.3 & 482.4 \\
$\mathrm{CO}_{3}^{2-}\left(\mathrm{mg} \mathrm{L}^{-1}\right)$ & 34.2 & 54.0 \\
$\mathrm{Cl}^{-}\left(\mathrm{mg} \mathrm{L}^{-1}\right)$ & 166.5 & 3571.7 \\
$\mathrm{NO}_{3}^{-}\left(\mathrm{mg} \mathrm{L}^{-1}\right)$ & 21.5 & 0.0 \\
$\mathrm{TOC}^{\left(\mathrm{mg} \mathrm{L}^{-1}\right)}$ & 13.2 & 125.1 \\
$\mathrm{COD}\left(\mathrm{mg} \mathrm{L}^{-1}\right)$ & 51.9 & 411.5 \\
\hline
\end{tabular}




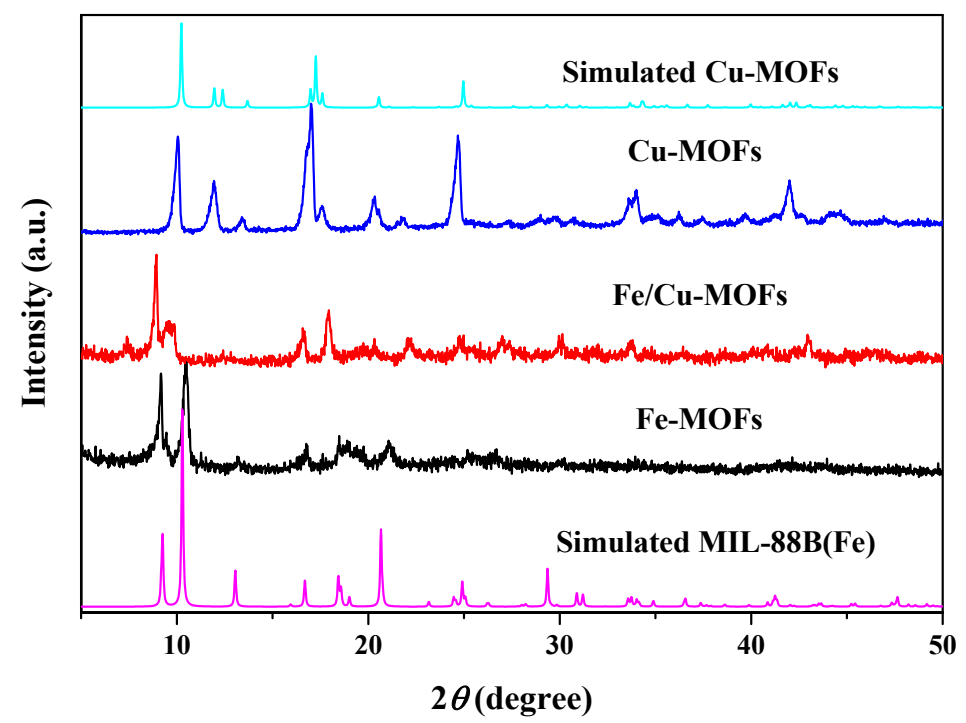

Fig. S1. XRD patterns of Fe-MOFs, $\mathrm{Fe} / \mathrm{Cu}-\mathrm{MOFs}$, and $\mathrm{Cu}-\mathrm{MOFs}$. 

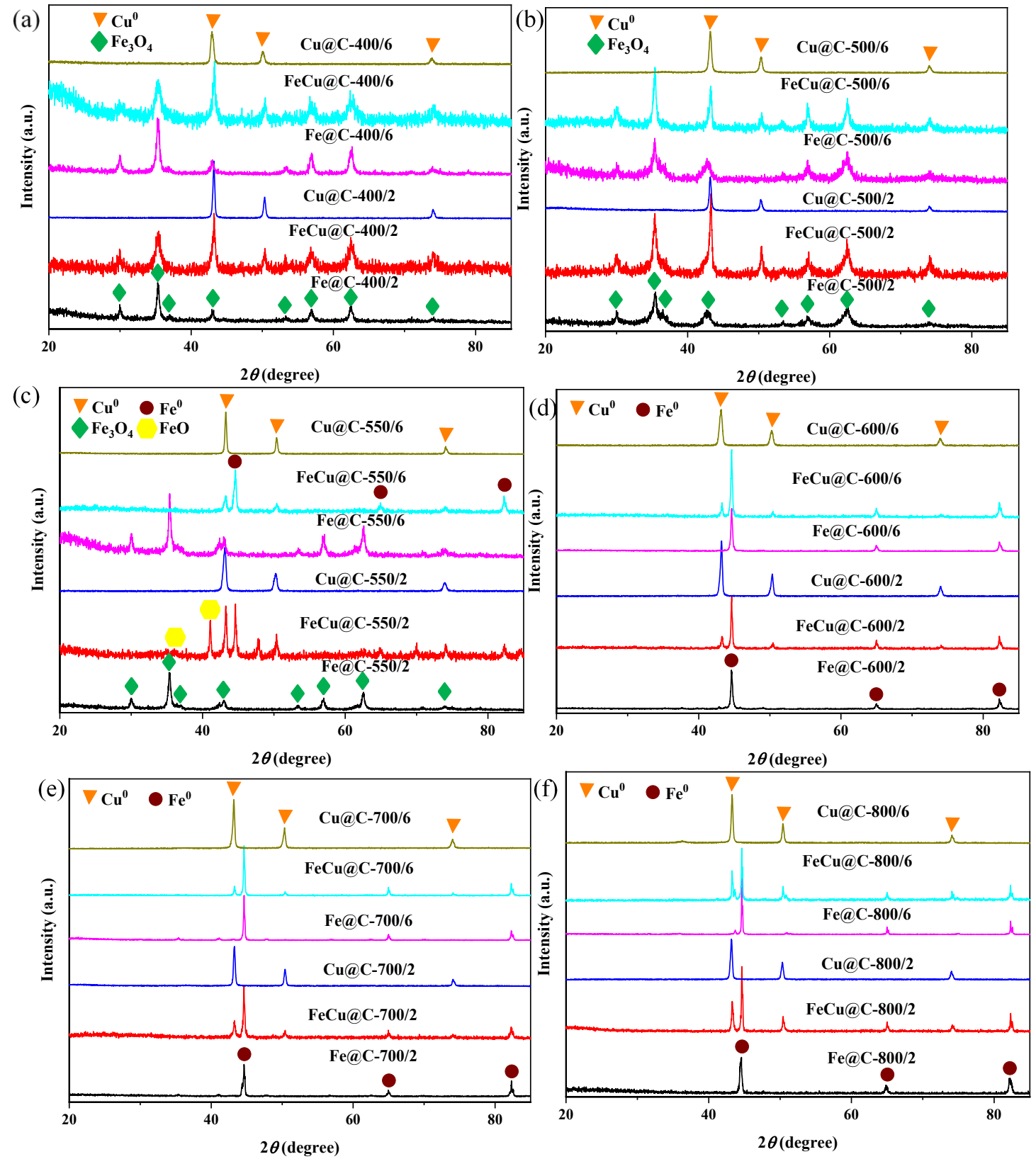

Fig. S2. XRD patterns of $\mathrm{Fe} @ \mathrm{PC}, \mathrm{FeCu} @ \mathrm{PC}$, and $\mathrm{Cu} @ \mathrm{PC}$ under different pyrolysis temperature: (a) $400^{\circ} \mathrm{C}$, (b) $500{ }^{\circ} \mathrm{C}$, (c) $550{ }^{\circ} \mathrm{C}$, (d) $600{ }^{\circ} \mathrm{C}$, (e) $700{ }^{\circ} \mathrm{C}$, and (f) $800^{\circ} \mathrm{C}$. 

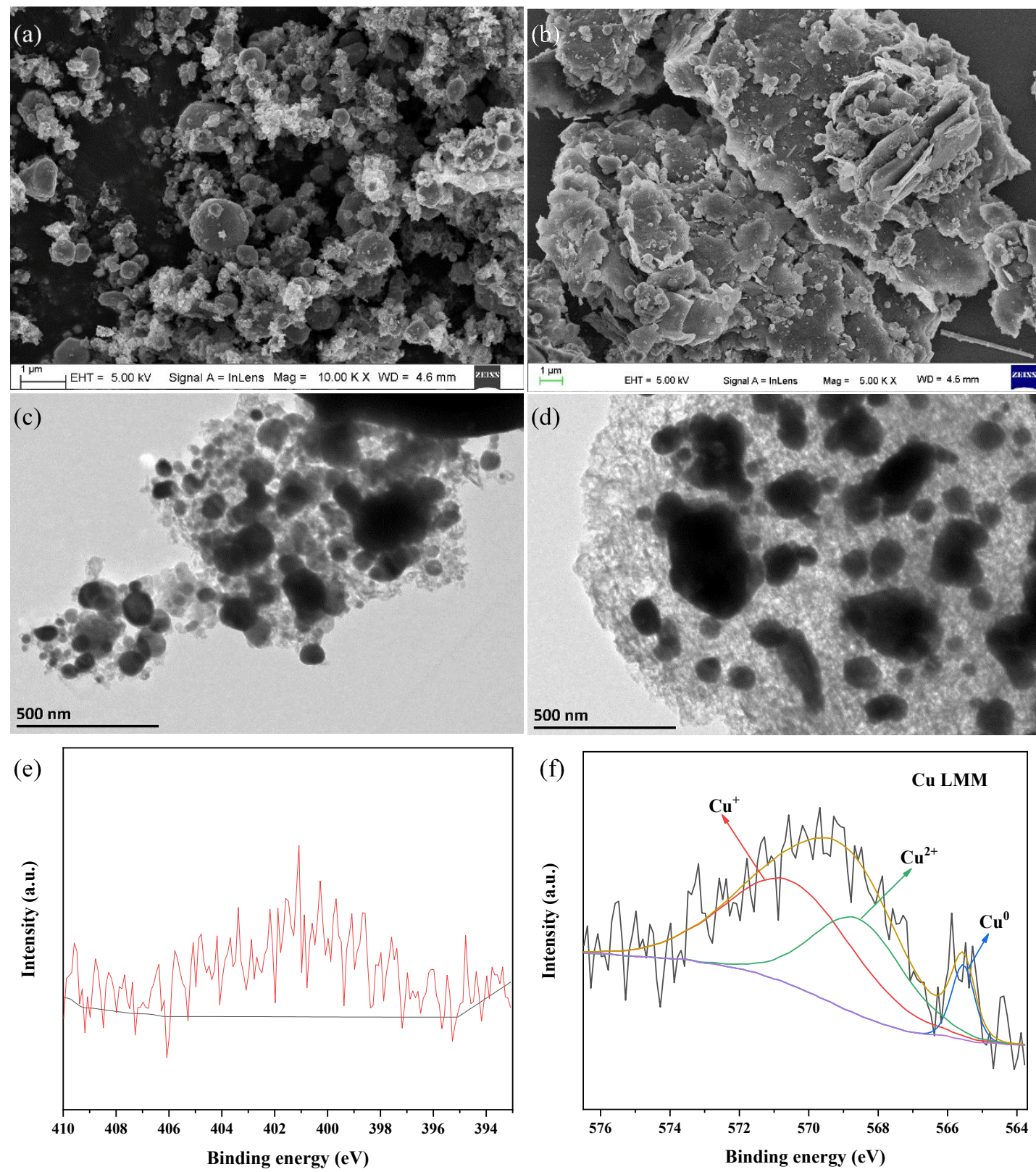

Fig. S3. SEM images of (a) Fe@PC-800/6 and (b) Cu@PC-800/6; TEM images of (c) Fe@PC-800/6 and (d) $\mathrm{Cu} @$ PC-800/6. (e) N 1s core-level spectra of FeCu@PC-800/6. (f) Cu LMM AES spectrum of 
FeCu@PC-800/6.

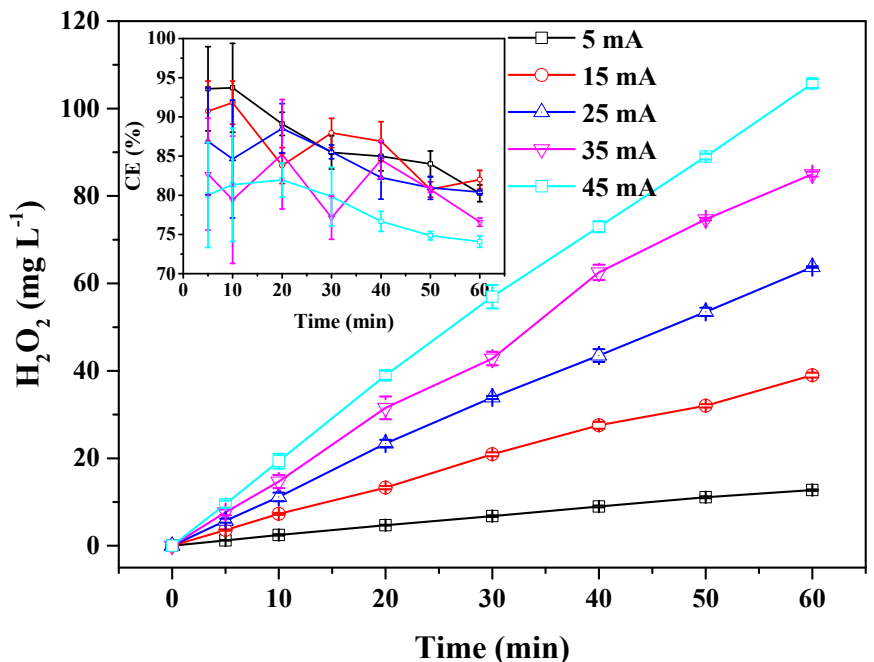

Fig. S4. Effect of current intensity on $\mathrm{H}_{2} \mathrm{O}_{2}$ production. Conditions: $200.0 \mathrm{~mL}, 0.05 \mathrm{M} \mathrm{Na}_{2} \mathrm{SO}_{4}, \mathrm{pH} 4.0$ The inset pattern is a photograph of the current efficiency of $\mathrm{H}_{2} \mathrm{O}_{2}$ production. 

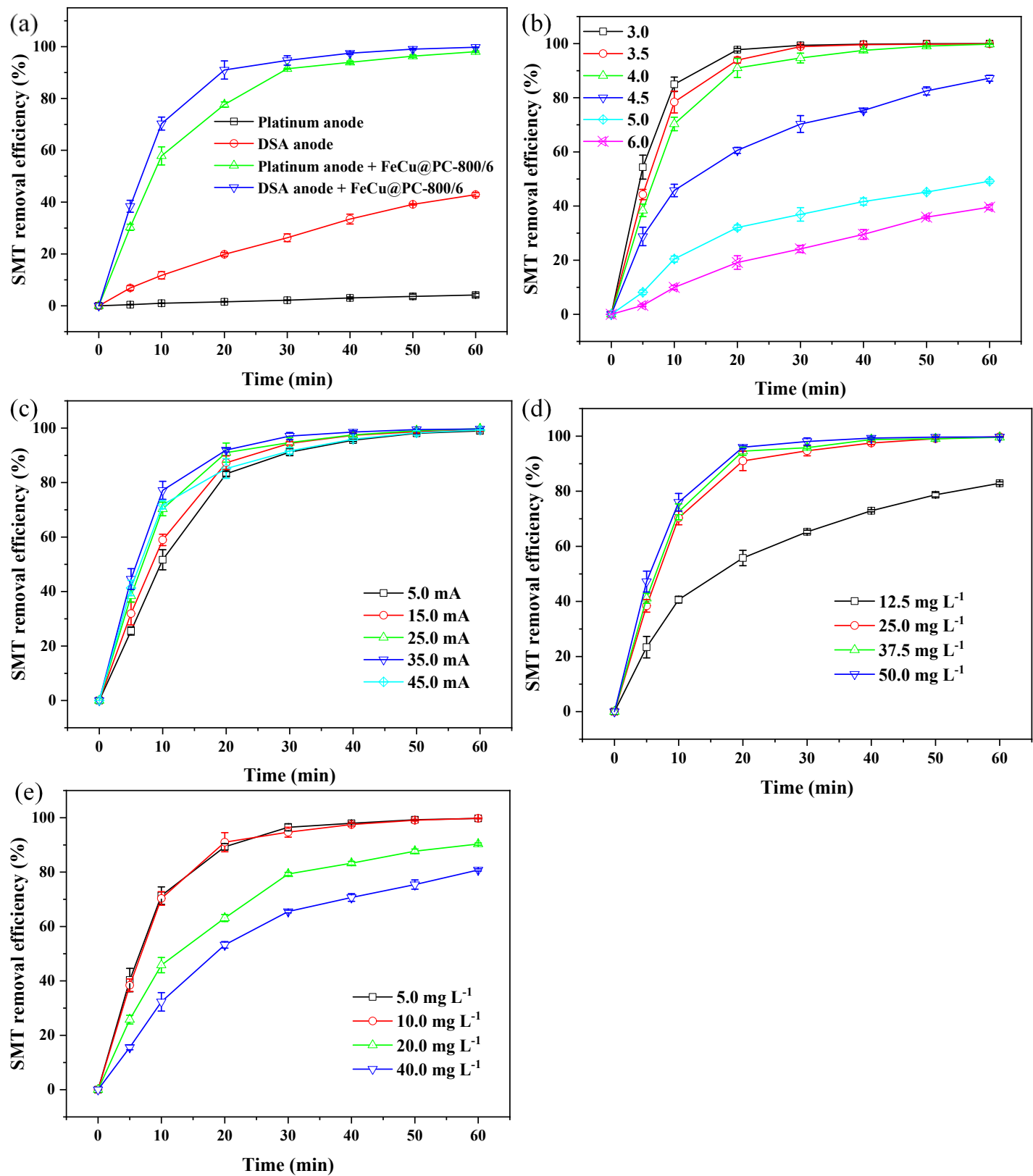

Fig. S5. The effect of various parameters on the SMT degradation in the FeCu@PC-800/6/Hetero-EF 
process: (a) anode, (b) initial $\mathrm{pH}$, (c) current, (d) catalyst concentration, and (e) SMT concentration. Except for the investigated parameter, other parameters were set as follows: $\mathrm{pH}, 4.0$; current, $25.0 \mathrm{~mA}$; catalyst, $25.0 \mathrm{mg} \mathrm{L}^{-1}$; initial SMT, $10.0 \mathrm{mg} \mathrm{L}^{-1}$.
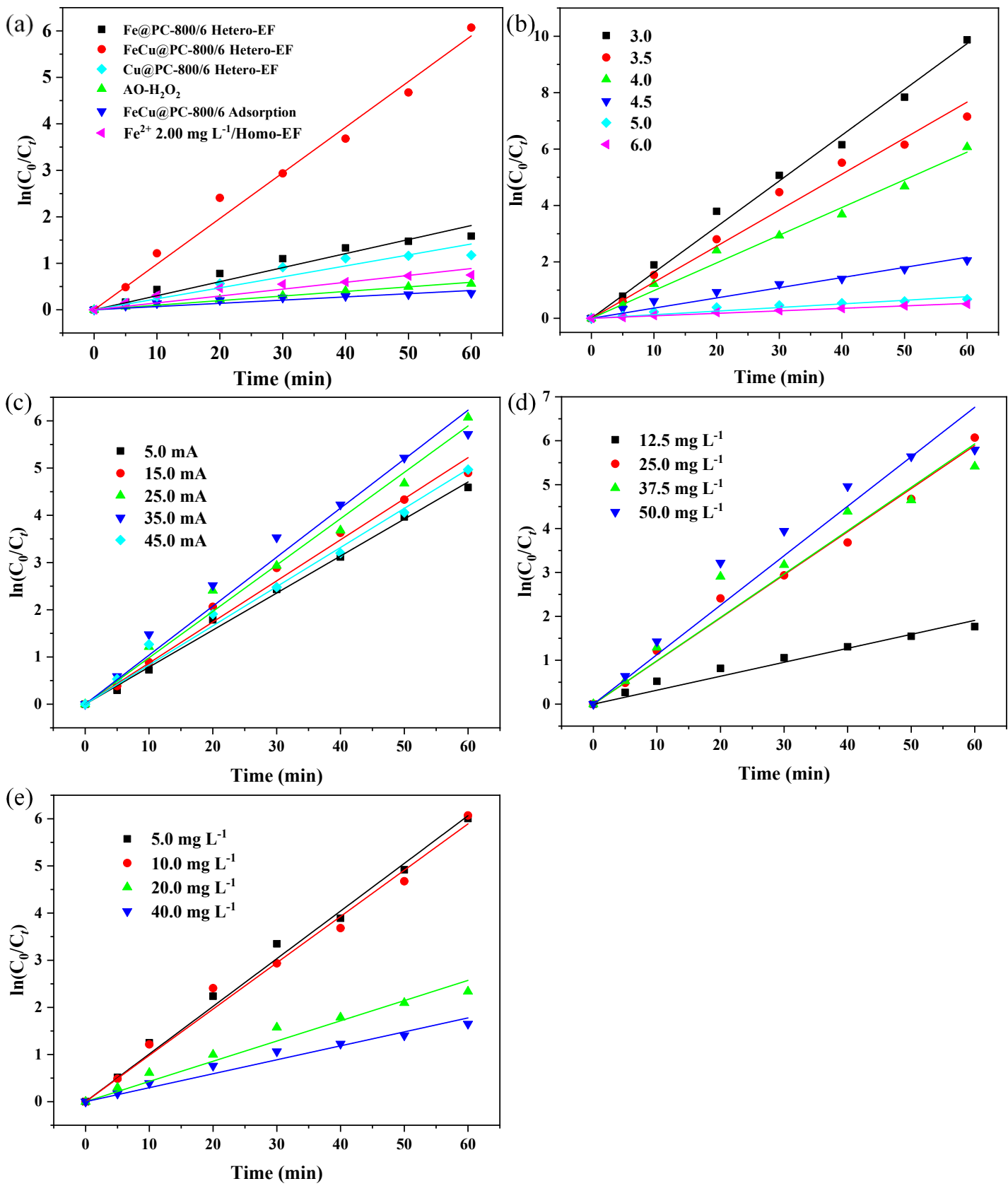
Fig. S6. The kinetics analysis as a pseudo-first-order reaction. (a) Comparison of the catalytic performance under different conditions. The effect of various parameters on the SMT degradation in the FeCu@PC-800/6/Hetero-EF process: (b) initial pH, (c) current, (d) catalyst concentration, and (e) SMT concentration.

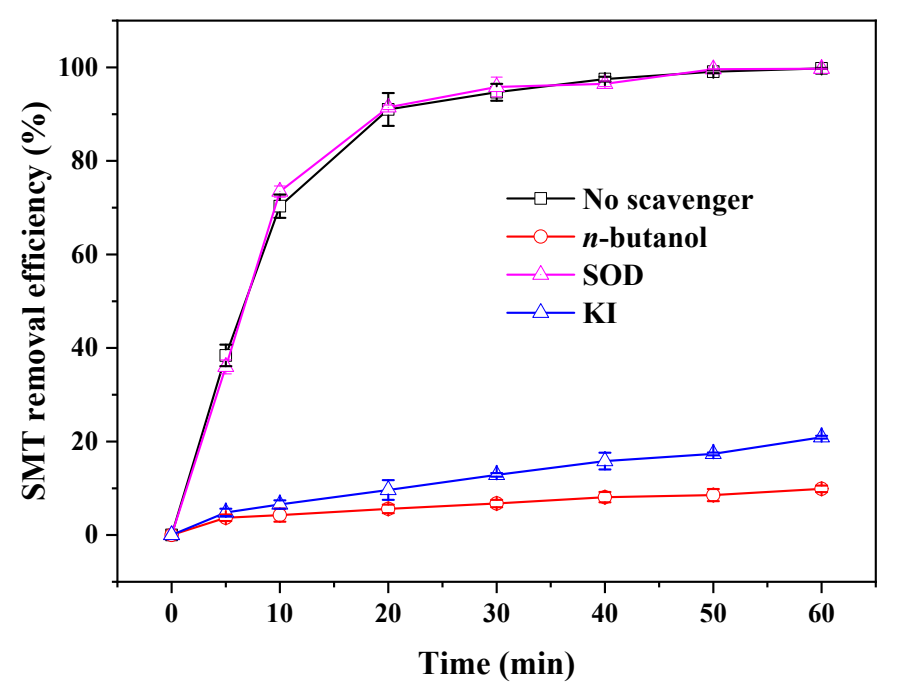

Fig. S7. Effect of scavengers: $300 \mathrm{mM}$-butanol, $3000 \mathrm{U} \mathrm{L}^{-1} \mathrm{SOD}$, and $10 \mathrm{mM} \mathrm{KI}$. Conditions: pH, 4.0; current, $25.0 \mathrm{~mA}$; initial SMT, $10.0 \mathrm{mg} \mathrm{L}^{-1}$; catalyst, $25.0 \mathrm{mg} \mathrm{L}^{-1}$. 


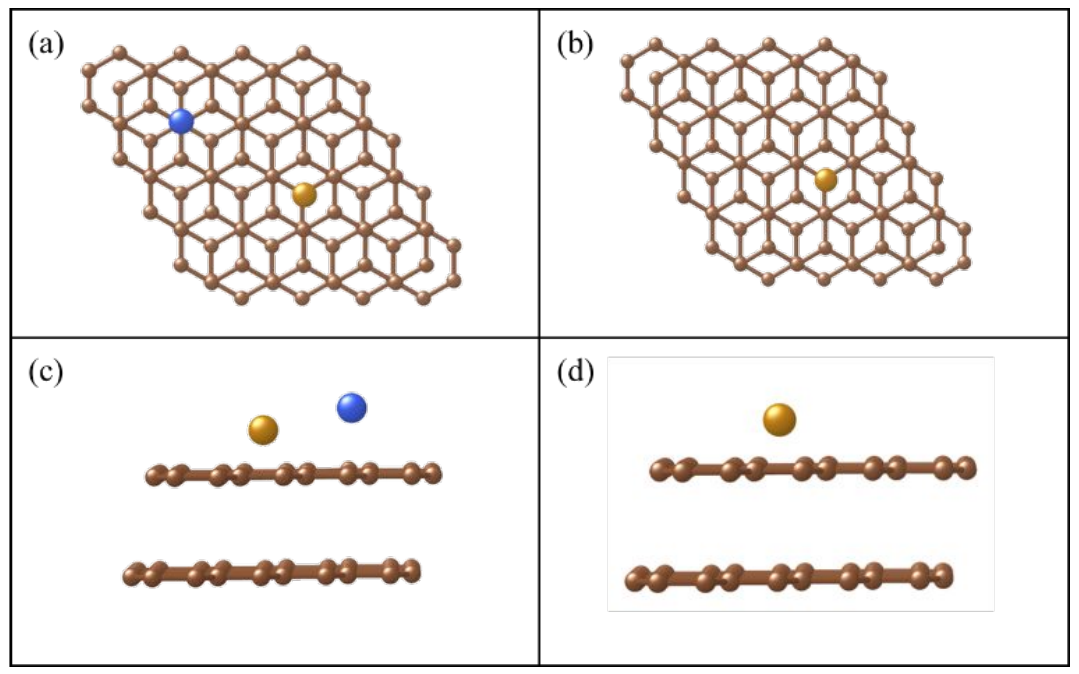

Fig. S8. Top-view and side-view structures of FeCu@PC-800/6 (a, c) and Fe@PC-800/6 (b, d). 


\begin{tabular}{|c|c|c|}
\hline PC & $\mathrm{Fe} @ \mathrm{PC}-800 / 6$ & $\mathrm{FeCu} @ \mathrm{PC}-800 / 6$ \\
\hline 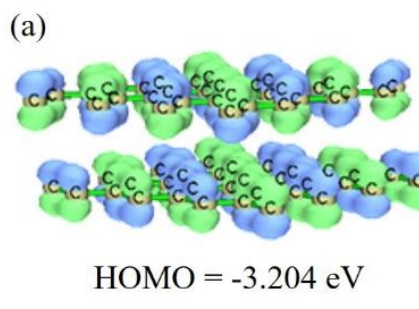 & 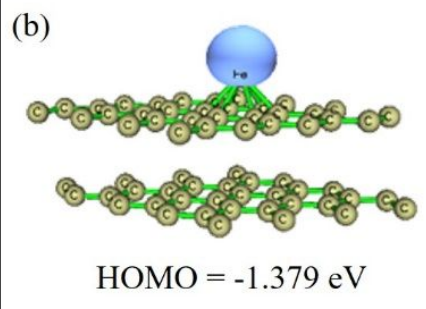 & 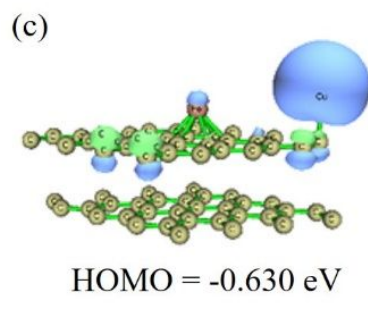 \\
\hline 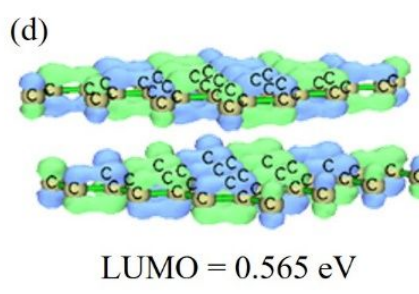 & 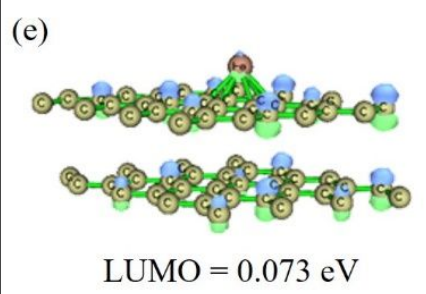 & 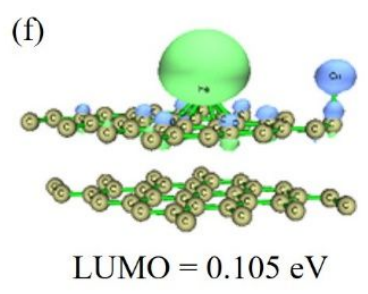 \\
\hline $\mathrm{Gap}_{\text {номо-LUмо }}=3.769 \mathrm{eV}$ & $\mathrm{Gap}_{\text {номо-LUмо }}=1.452 \mathrm{eV}$ & $\mathrm{Gap}_{\text {HОмО-LUмо }}=0.735 \mathrm{eV}$ \\
\hline
\end{tabular}

Fig. S9. HOMO and LUMO spatial distribution of PC (a, d), Fe@PC-800/6 (b, e) and FeCu@PC-800/6 (c, f) respectively. (vaspmo03 and mutiwfn software deal with this picture, and green and blue regions represents the positive and negative electron cloud, respectively.) 


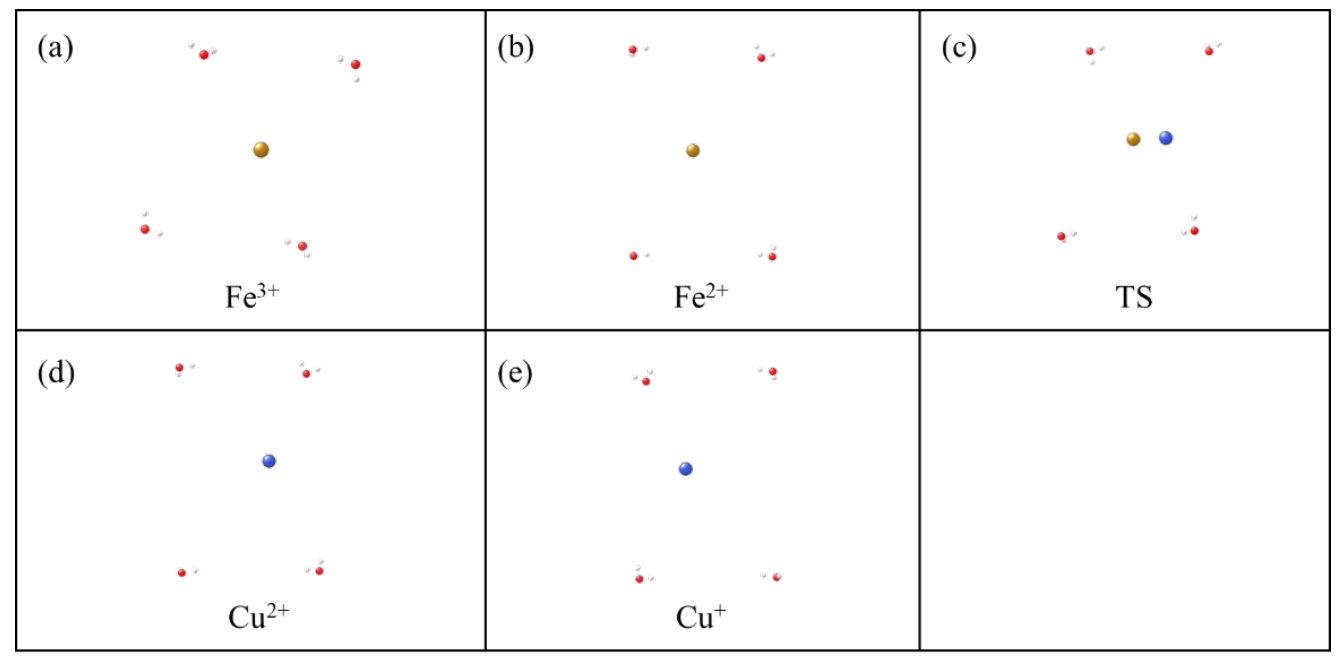

Fig. S10. The model of hydrated ion. 


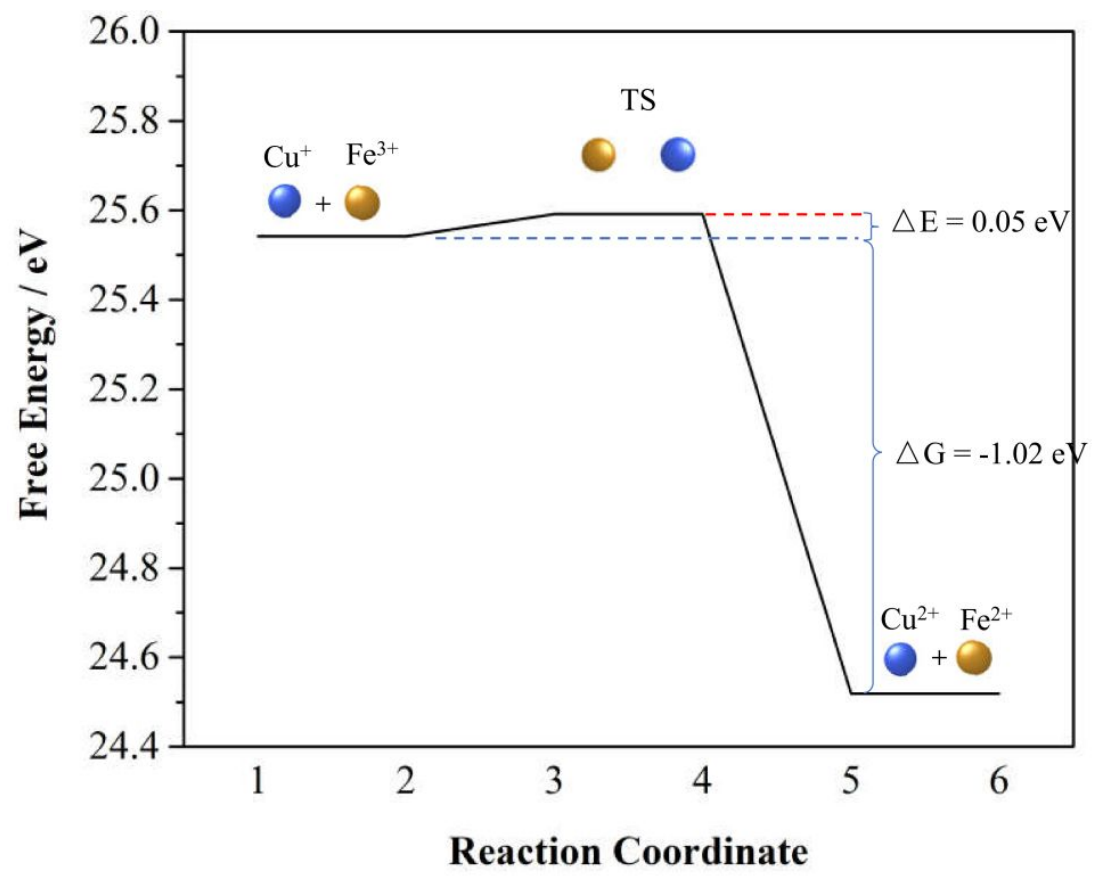

Fig. S11. The reaction energies of $\mathrm{Cu}^{+}$reducing $\mathrm{Fe}^{3+}$ in solution. 


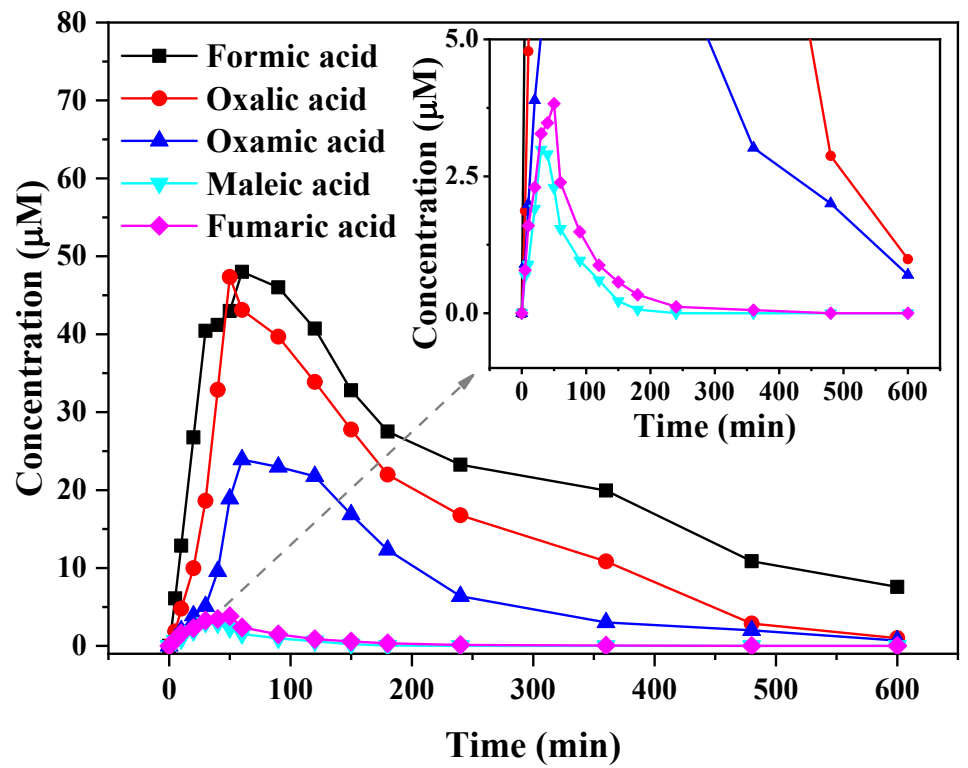

Fig. S12. Evolution of carboxylic acids formed in the FeCu@PC-800/6/Hetero-EF process. Conditions: initial SMT, $50.0 \mathrm{mg} \mathrm{L}^{-1} ; \mathrm{pH}, 4.0$; catalyst, $50.0 \mathrm{mg} \mathrm{L}^{-1}$; current, $25.0 \mathrm{~mA}$. 
(a)

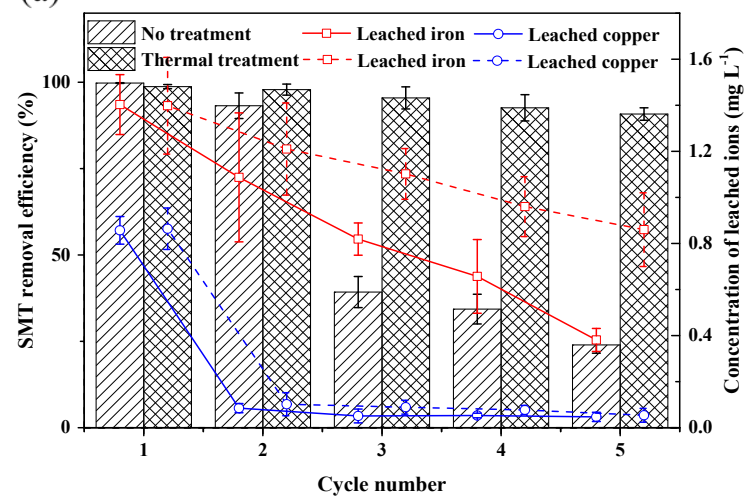

(b)

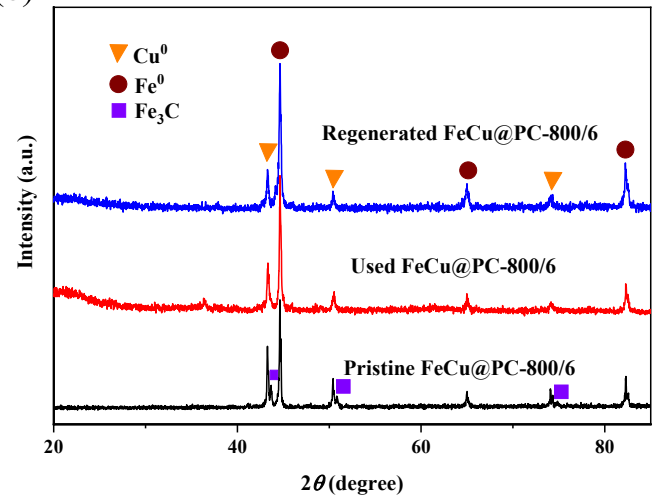

Fig. S13. (a) Consecutive runs to probe the evolution of catalytic activity for $\mathrm{FeCu} @ \mathrm{PC}-800 / 6$ as well as the corresponding iron and copper ions concentration. (For the cycling tests, the catalyst was recycled by external magnetic field, and then directly reused for the next run without washing. The thermal treatment on the recycled catalyst at $400{ }^{\circ} \mathrm{C}$ for $1 \mathrm{~h}$ in $\mathrm{H}_{2}$ with the heating rate of $10^{\circ} \mathrm{C} \mathrm{min}^{-1}$ was applied to regenerate the catalytic activity before each run.) Conditions: $\mathrm{pH}, 4.0$; current, $25.0 \mathrm{~mA}$; initial SMT, $10.0 \mathrm{mg} \mathrm{L}^{-1}$; catalyst, $25.0 \mathrm{mg} \mathrm{L}^{-1}$. (b) XRD pattern of pristine, used and regenerated $\mathrm{FeCu} @ \mathrm{PC}-800 / 6$. 

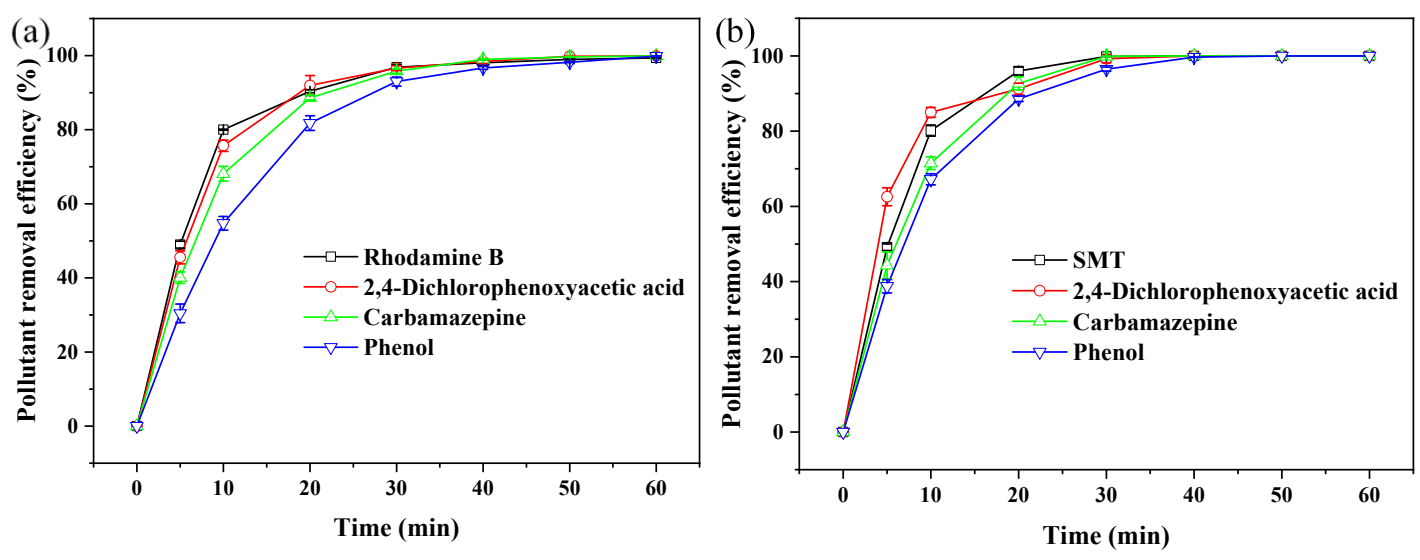

Fig. S14. (a) Degradation of rhodamine B, 2,4-dichlorophenoxyacetic acid, carbamazepine, and phenol, respectively, in the $\mathrm{FeCu} @ \mathrm{PC}-800 / 6 /$ Hetero-EF process. Conditions: pH, 4.0; current, $25.0 \mathrm{~mA}$; pollutant, $10.0 \mathrm{mg} \mathrm{L}^{-1}$; catalyst, $25.0 \mathrm{mg} \mathrm{L}^{-1}$. (b) Degradation of the mixture of rhodamine $\mathrm{B}, 2$,4dichlorophenoxyacetic acid, carbamazepine, and phenol in the FeCu@PC-800/6/Hetero-EF process. Conditions: $\mathrm{pH}, 4.0$; current, $25.0 \mathrm{~mA}$; each pollutant, $1.0 \mathrm{mg} \mathrm{L}^{-1}$; catalyst, $25.0 \mathrm{mg} \mathrm{L}^{-1}$. 

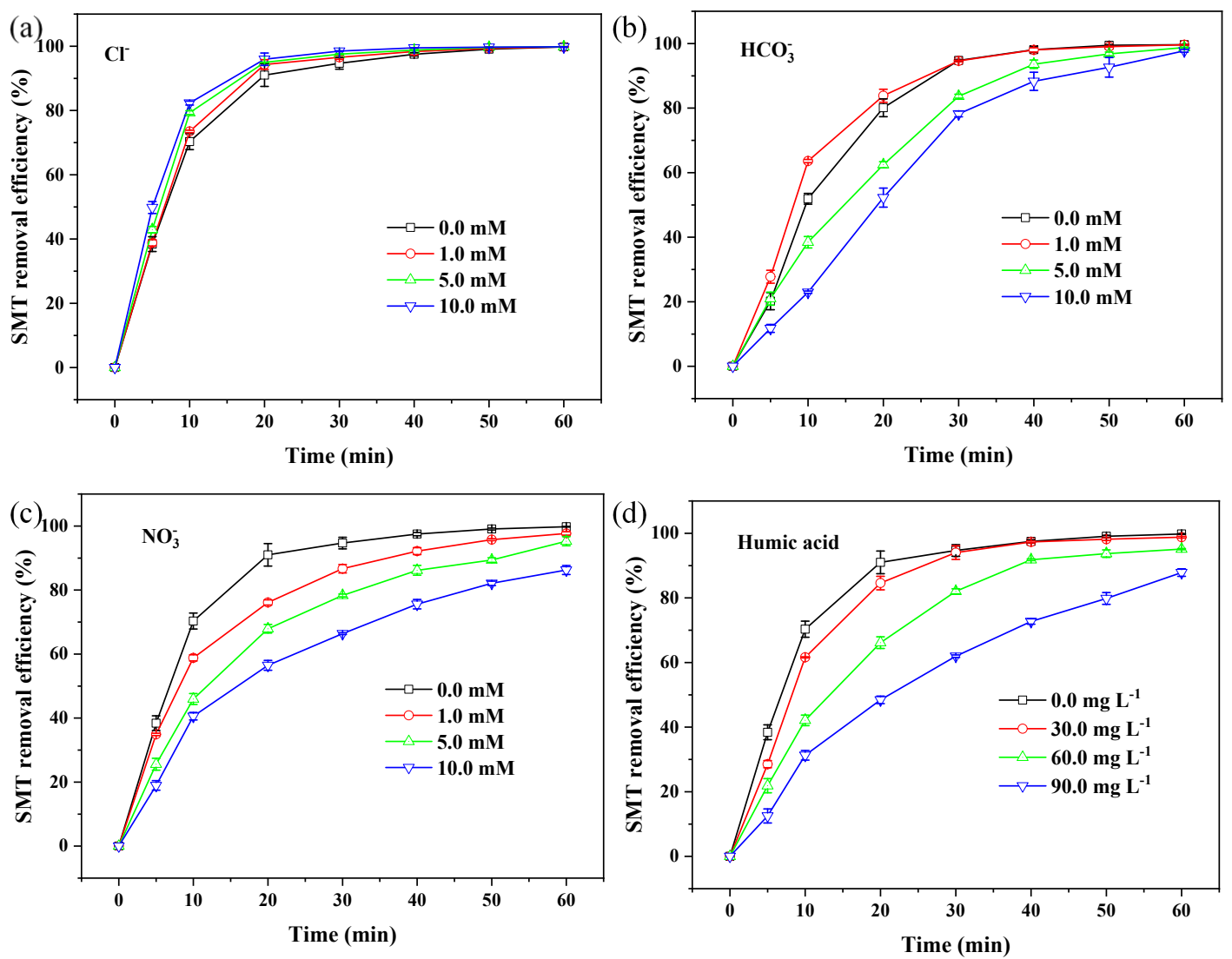

Fig. S15. Effect of background constituents: (a) $\mathrm{NaCl}$, (b) $\mathrm{NaHCO}_{3}$, (c) $\mathrm{NaNO}_{3}$ and (d) humic acid. Conditions: $\mathrm{pH}, 4.0$; current, $25.0 \mathrm{~mA}$; SMT, $10.0 \mathrm{mg} \mathrm{L}^{-1}$; catalyst, $25.0 \mathrm{mg} \mathrm{L}^{-1}$. 


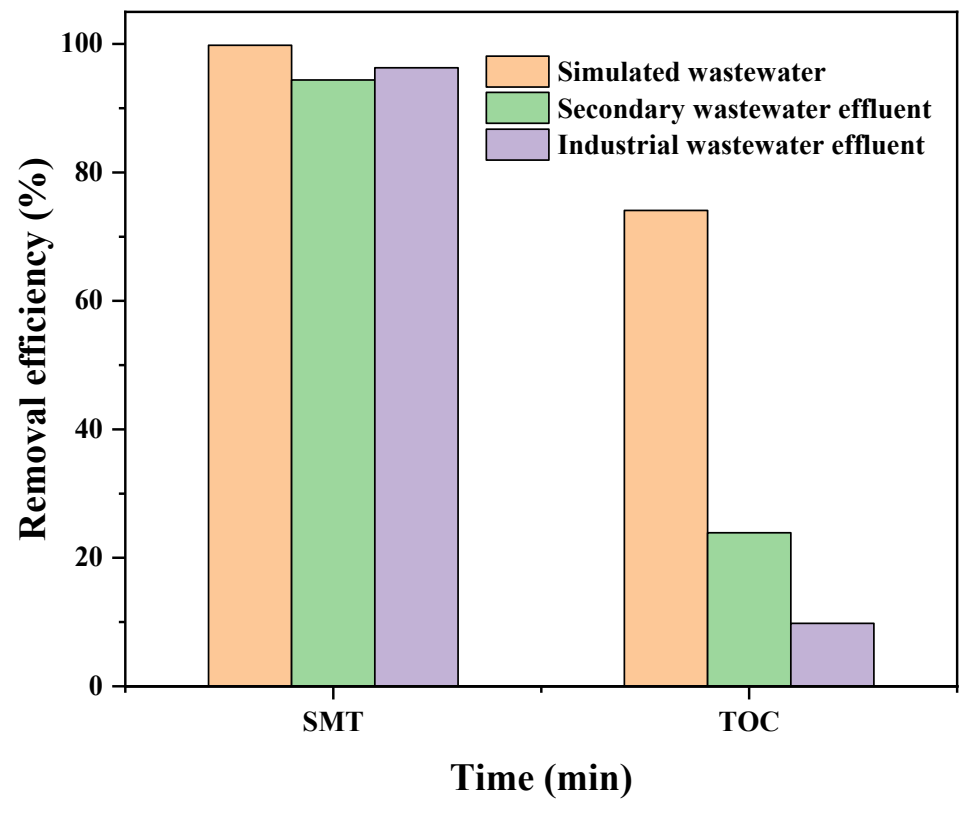

Fig. S16. Degradation of SMT in the simulated wastewater, secondary wastewater or industrial wastewater effluent by the $\mathrm{FeCu} @$ PC-800/6/Hetero-EF process. Conditions: pH, 4.0; current, 25.0 mA; SMT, $10.0 \mathrm{mg} \mathrm{L}^{-1}$; catalyst, $25.0 \mathrm{mg} \mathrm{L}^{-1}$. 

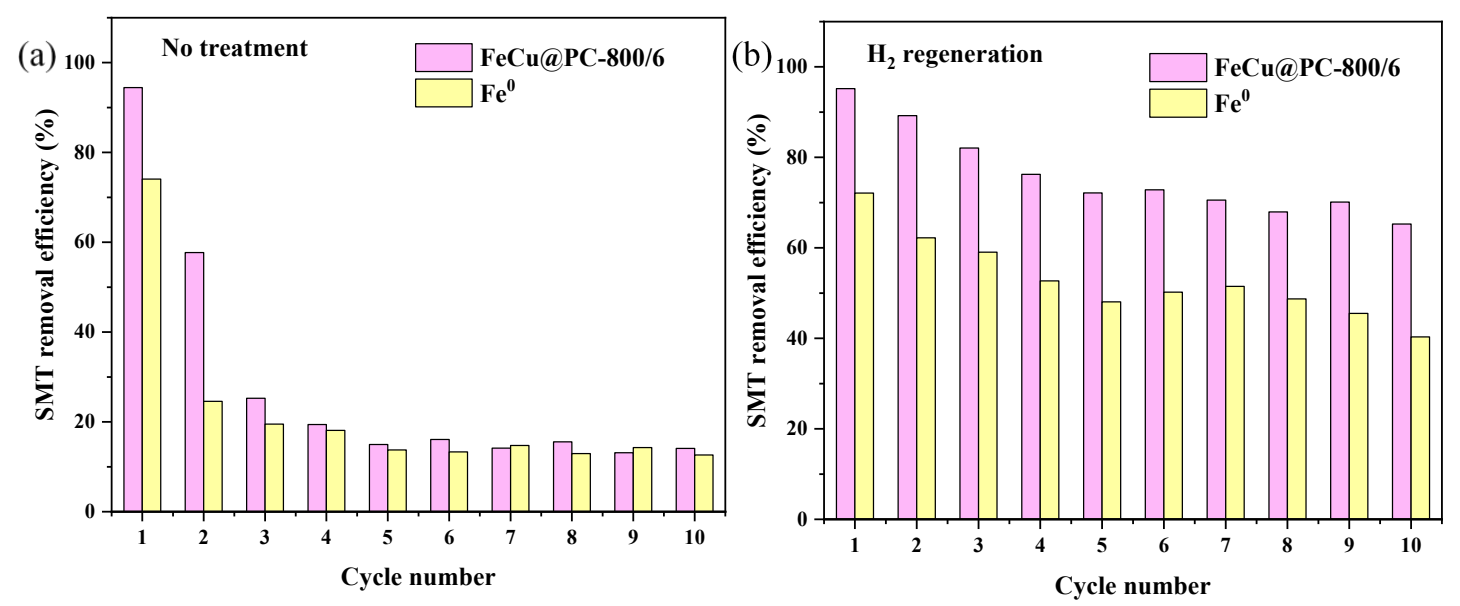

Fig. S17. (a) Consecutive runs to probe the evolution of catalytic activity for $\mathrm{FeCu} @ \mathrm{PC}-800 / 6$ and $\mathrm{Fe}^{0}$ in the secondary wastewater effluent. (For cycling tests, the catalyst was recycled by external magnetic field, and then directly reused for the next run without washing.) (b) After regeneration, probe the evolution of catalytic activity for $\mathrm{FeCu} @ \mathrm{PC}-800 / 6$ and $\mathrm{Fe}^{0}$ in the secondary wastewater effluent. (The thermal treatment on the recycled catalyst at $400{ }^{\circ} \mathrm{C}$ for $1 \mathrm{~h}$ in $\mathrm{H}_{2}$ with the heating rate of $10{ }^{\circ} \mathrm{C} \mathrm{min}^{-1}$ was applied to regenerate the catalytic activity before each run.) Conditions: $\mathrm{pH}, 4.0$; current, $25.0 \mathrm{~mA}$; SMT, $10.0 \mathrm{mg} \mathrm{L}^{-1}$; catalyst, $25.0 \mathrm{mg} \mathrm{L}^{-1}$. 


\section{References}

(1) Kresse, G.; Furthmüller, J. Software VASP, vienna (1999). Phys. Rev. B 1996, 54 (11), 169.

(2) Yang, Y.; Xu, L.; Li, W.; Fan, W.; Song, S.; Yang, J. Adsorption and degradation of sulfadiazine over nanoscale zero-valent iron encapsulated in three-dimensional graphene network through oxygendriven heterogeneous Fenton-like reactions. Appl. Catal. B: Environ. 2019, 259, 118057.

(3) Xu, L.; Yang, Y.; Li, W.; Tao, Y.; Sui, Z.; Song, S.; Yang, J. Three-dimensional macroporous graphene-wrapped zero-valent copper nanoparticles as efficient micro-electrolysis-promoted Fenton-like catalysts for metronidazole removal. Sci. Total Environ. 2019, 658, 219-233.

(4) Zhang, X.; Ding, Y.; Tang, H.; Han, X.; Zhu, L.; Wang, N. Degradation of bisphenol A by hydrogen peroxide activated with $\mathrm{CuFeO}_{2}$ microparticles as a heterogeneous Fenton-like catalyst: efficiency, stability and mechanism. Chem. Eng. J. 2014, 236, 251-262.

(5) Guimaraes, I. R.; Giroto, A.; Oliveira, L. C.; Guerreiro, M. C.; Lima, D. Q.; Fabris, J. D. Synthesis and thermal treatment of $\mathrm{Cu}$-doped goethite: Oxidation of quinoline through heterogeneous fenton process. Appl. Catal. B: Environ. 2009, 91 (3-4), 581-586.

(6) Yamaguchi, R.; Kurosu, S.; Suzuki, M.; Kawase, Y. Hydroxyl radical generation by zero-valent iron/ $\mathrm{Cu}(\mathrm{ZVI} / \mathrm{Cu})$ bimetallic catalyst in wastewater treatment: Heterogeneous Fenton/Fenton-like reactions by Fenton reagents formed in-situ under oxic conditions. Chem. Eng. J. 2018, 334, 1537-1549. (7) Karthikeyan, S.; Pachamuthu, M.; Isaacs, M. A.; Kumar, S.; Lee, A. F.; Sekaran, G. Cu and Fe oxides dispersed on SBA-15: A Fenton type bimetallic catalyst for N, N-diethyl-p-phenyl diamine degradation. Appl. Catal. B: Environ. 2016, 199, 323-330.

(8) Zheng, C.; Yang, C.; Cheng, X.; Xu, S.; Fan, Z.; Wang, G.; Wang, S.; Guan, X.; Sun, X. Specifically enhancement of heterogeneous Fenton-like degradation activities for ofloxacin with synergetic effects of bimetallic Fe-Cu on ordered mesoporous silicon. Sep. Purif. Technol. 2017, 189, 357-365.

(9) Wang, J.; Liu, C.; Hussain, I.; Li, C.; Li, J.; Sun, X.; Shen, J.; Han, W.; Wang, L. Iron-copper bimetallic nanoparticles supported on hollow mesoporous silica spheres: the effect of $\mathrm{Fe} / \mathrm{Cu}$ ratio on heterogeneous Fenton degradation of a dye. RSC Adv. 2016, 6 (59), 54623-54635.

(10) Xia, M.; Long, M.; Yang, Y.; Chen, C.; Cai, W.; Zhou, B. A highly active bimetallic oxides catalyst 
supported on Al-containing MCM-41 for Fenton oxidation of phenol solution. Appl. Catal. B: Environ. 2011, 110, 118-125.

(11) Ling, Y.; Long, M.; Hu, P.; Chen, Y.; Huang, J. Magnetically separable core-shell structural $\gamma$ $\mathrm{Fe}_{2} \mathrm{O}_{3} @ \mathrm{Cu} / \mathrm{Al}-\mathrm{MCM}-41$ nanocomposite and its performance in heterogeneous Fenton catalysis. $J$. Hazard. Mater. 2014, 264, 195-202.

(12) Sun, Y.; Yang, Z.; Tian, P.; Sheng, Y.; Xu, J.; Han, Y.-F. Oxidative degradation of nitrobenzene by a Fenton-like reaction with Fe-Cu bimetallic catalysts. Appl. Catal. B: Environ. 2019, 244, 1-10.

(13) Dükkanc1, M.; Gündüz, G.; Yılmaz, S.; Prihod'Ko, R. Heterogeneous Fenton-like degradation of Rhodamine $6 \mathrm{G}$ in water using CuFeZSM-5 zeolite catalyst prepared by hydrothermal synthesis. $J$. Hazard Mater. 2010, 181 (1-3), 343-350.

(14) Wang, Y.; Zhao, H.; Zhao, G. Iron-copper bimetallic nanoparticles embedded within ordered mesoporous carbon as effective and stable heterogeneous Fenton catalyst for the degradation of organic contaminants. Appl. Catal. B: Environ. 2015, 164, 396-406.

(15) Tang, J.; Wang, J. MOF-derived three-dimensional flower-like FeCu@C composite as an efficient Fenton-like catalyst for sulfamethazine degradation. Chem. Eng. J. 2019, 375, 122007.

(16) Xu, J.; Li, Y.; Yuan, B.; Shen, C.; Fu, M.; Cui, H.; Sun, W. Large scale preparation of Cu-doped $\alpha$ $\mathrm{FeOOH}$ nanoflowers and their photo-Fenton-like catalytic degradation of diclofenac sodium. Chem. Eng. J. 2016, 291, 174-183.

(17) Li, K.; Zhao, Y.; Janik, M. J.; Song, C.; Guo, X. Facile preparation of magnetic mesoporous $\mathrm{Fe}_{3} \mathrm{O}_{4} / \mathrm{C} / \mathrm{Cu}$ composites as high performance Fenton-like catalysts. Appl. Surf. Sci. 2017, 396, 1383-1392. (18) Zhao, H.; Qian, L.; Guan, X.; Wu, D.; Zhao, G. Continuous bulk FeCuC aerogel with ultradispersed metal nanoparticles: an efficient 3D heterogeneous electro-Fenton cathode over a wide range of $\mathrm{pH}$ 3-9. Environ. Sci. Technol. 2016, 50 (10), 5225-5233.

(19) Barros, W. R.; Steter, J. R.; Lanza, M. R.; Tavares, A. C. Catalytic activity of $\mathrm{Fe}_{3-\mathrm{x}} \mathrm{Cu}_{\mathrm{x}} \mathrm{O} 4(0 \leq \mathrm{x} \leq$ $0.25)$ nanoparticles for the degradation of Amaranth food dye by heterogeneous electro-Fenton process. Appl. Catal. B: Environ. 2016, 180, 434-441. 